pre-operative pain in these cases may well be due to secondary infection, but the persisting post-operative pain, occurring even in a widely laid open cavity, is inexplicable. It cannot be due to direct involvement of the dura, as the dura over the temporal bone is not a site from which painful stimuli can be elicited (Wolff, 1948). Nevertheless, in Cases 2, 3, and 4, in which pain was a dominant symptom, the dura was invaded by the growth, whereas the pain disappeared after operation in Cases 1 and 5, in which there was no dural involvement. We are not prepared to offer any explanation of these observations. Pain is a common symptom in cancer, and its cause is often obscure.

2. Chronic Suppuration.-A history of genuine chronic suppuration was obtained in only two of our six cases. In the other cases the otorrhoea could be attributed to the presence of tumour rather than to pre-existing suppuration.

3. Facial Paralysis.-This was a presenting symptom in two cases, and was present but unnoticed by the patient in one case.

4. A Blood-stained Aural Discharge.-This was present in four out of the five cases.

5. Sequestration of Bone.-This was present in one case.

6. Post-aural Swelling.-This was present in one case.

7. Metastasis.-Neighbouring or distant metastasis has rarely been reported in the literature, but it occurred in Case 6. Whether the method of treatment adopted was the correct one remains to be seen, but the result so far has been satisfactory.

From our experience it seems that pain and the presence of a blood-stained discharge, particularly if there is also a facial palsy, are the most reliable indications of malignant disease in the middle ear. The diagnosis must be clinched by biopsy.

\section{Cause of Death}

The cause of death is usually given as malignant cachexia. In some cases invasion of the meninges leads to fatal intracranial complications. In others a fatal haemorrhage occurs from erosion of the jugular bulb or the carotid artery. Death from a distant metastasis has not been recorded.

In our series, one patient died from coronary occlusion and two from direct intracranial spread of the disease.

\section{Treatment}

In so far as so rare a disease can be said to have any settled mode of treatment, it would be a combination of surgery and radiotherapy. One of our cases received radiotherapy before surgery, whilst the others were treated by post-operative irradiation (at the Royal Cancer Hospital, London, under the direction of Dr. M. Lederman). The extent of the operation varied with the extent of the discase. As in all cancer surgery, no anatomical boundaries were respected, and no attempt was made to preserve any involved structure which could be excised.

For instance, in most cases the inner ear was removed in whole or in part; in two cases considerable portions of the capsule of the temporo-mandibular joint were excised; and in one case the pinna was sacrificed. When the region of the facial nerve was involved in growth no attempt to preserve the nerve was made at the expense of a skimped excision.

However, in Cases 2, 3, and 4, pursuing the disease anteriorly would have meant excision of the temporomandibular joint and the adjacent part of the base of the skull, with no certainty, even then, of reaching the limits of the growth. In the light of subsequent events, this is probably the course which should have been followed to give the patient the best possible chance, as it seems clear that radiotherapy is unavailing in growth so deeply buried in bone. In Case 3 the whole bony meatus and the parotid gland should perhaps also have been excised in order to comply with the essential requirements of tumour surgery. These are speculative suggestions, offered after reflecting on the clinical course of these few cases, and applying general surgical opinions which are commonly accepted. The tumours are not highly malignant, and distant metastasis is almost unknown. The problem, then, is entirely one of adequate local removal, and this seemingly applies particularly to the bone, as irradiation appears to be effective in controlling the soft-tissue invasion. Before embarking on any case a clear idea must be entertained of the possible directions of spread in the bone and a feasible means of excising these areas. Such excisions are not insurmountable technical problems, provided they are not presented suddenly in the course of an operation conceived in lesser terms.

\section{Prognosis}

The prognosis of middle-ear carcinoma is very poor indeed. Only one case is to be found in the literature in which the patient has survived for more than five years. In this series six cases are reported; one patient is alive and well six and a half years after coming under observation, and one other, treated a year ago, was also alive and without any evidence of recurrence at the time of writing. In both these cases the disease did not involve the dura, which is the factor enormously worsening the outlook.

\section{Summary}

Six cases of carcinoma of the middle ear are described. Suggestions for more radical surgical excision are offered.

REFERENCES

Bowman, R. J. (1940). Ann. Otol., St. Louis, 49, 225 Furstenberg, A. C. (1924), Ibid., 33, 677.

Risch, O. C., and Lisa, J. R. (1938). Laryngoscope, 48, 668

Wolff, H. G. (1948). Headache and other Head Pain, p. 66. Oxford Univ. Press, New York.

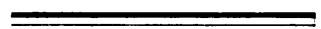

\section{ANGULAR STOMATITIS AND ITS ASSOCIATION WITH ARTIFICIAL DENTURES}

BY

J. G. HARKNESS, M.B., Ch.B.

Registrar in the Department of Dermatology, the General Infirmary at Leeds

Angular stomatitis is seen by the general physician as evidence of nutritional (especially vitamin B) deficiency or of anaemia; but such patients rarely visit the dermatologist. Nevertheless, many patients suffering from angular stomatitis are seen in dermatological and dental departments, and few of them are found to be suffering from general nutritional disease. Most of these patients show a local infective cause for the condition or have some dental fault or both.

The series of patients reviewed in this paper were drawn from the dermatology department of the General Infirmary at Leeds, and the work was done in conjunction with the staff of the dental school.

The literature on the subject is vague; but Vickers (1949) made an important contribution, and his approach has been followed.

There appears to be little doubt that there are several aetiological factors, and in some cases more than one of these may be contributory, so that careful consideration of each particular patient is necessary if treatment is to be successful. Generally accepted aetiological factors include : (1) infection; (2) ariboflavinosis ; (3) generalized seborrhoeic or flexural eczema; (4) sensitization to dental materials; and (5) malocclusion. 


\section{Infection}

Angular stomatitis may occur as a manifestation of lues in the secondary or tertiary stages. Other infections, especially of the respiratory or gastro-intestinal tract, may also cause the condition, including moniliasis. In Case 14 attacks of angular stomatitis were coincidental with recurrent tonsillitis, and in Case 16 relapses always followed a common cold.

\section{Ariboflavinosis}

Although in this series ariboflavinosis does not seem to be important, this may be explained by the direction of such cases to general medical rather than dermatological clinics. Blood vitamin levels were not undertaken as a routine; but all patients were questioned closely with a view to establishing possible dietetic deficiency or finding evidence of a malabsorptive syndrome : Case 23 proved interesting in this respect. This patient suffered from Crohn's disease for many years, and her stomatitis cleared soon after operation for this condition and has not recurred. Her case was also interesting in that there appeared to be a familial tendency to angular stomatitis, as both her brother and her sister (Case 12) suffered from the condition, though neither gave any similar history suggestive of malabsorption.

\section{Generalized Seborrhoeic or Flexural Eczema}

Angular stomatitis often occurs as part of a generalized seborrhoeic or flexural eczema and clears or relapses with the general condition; but it may occur as the sole manifestation.

This condition involves more of the skin adjacent to the angles of the mouth than usual and proves very resistant to treatment and liable to relapse readily. An example of this type is Case 17, which proved extremely resistant to various local measures, including antibiotics, dental treatment, and to $x$-ray therapy, but cleared on the administration of ung. steroxin and had not relapsed six months later.

\section{Sensitization to Denture Materials}

Some patients become sensitized to the material of their dentures, and in such cases the condition usually clears with the substitution of a different denture material. In most instances of sensitivity to denture material an area of hyperaemia, and in some cases actual oedema of the underlying mucous membrane, is present; but in three patients (Cases 1,6 , and 11) there were no mucosal changes, although patch tests to denture material were positive. Further, 12 patients (Cases 4, 8, 12, 18, 21, 26, 28, 32, 36, 41,43 , and 45) with mucosal changes were negative to patch tests. It is noteworthy that acrylic dentures were concerned in all these cases except one. As a control, colleagues at the dental school examined 100 random cases in the prosthetics department, and in only one of these was there any definite mucosal lesion without accompanying angular stomatitis or complaint of soreness of the gums. These people had been wearing dentures for from one year to forty years.

\section{Patch Testing}

This means of investigation of the presence of sensitivity was used, the method followed being that of Vickersnamely, the application of scrapings from the patients' own dentures to the skin, held in place by adhesive plaster. In several instances positive patch-test reactions were delayed, and it would seem advisable to retain the material in position for at least 96 hours, with a preliminary reading at 48 hours. Cases 1, 2, 3, 24, 33, and 44 demonstrate this delayed reaction.

It might well be argued that these delayed positive reactions could be due to mechanical friction or chemical action; but a control series of 20 medical students failed to produce any such mechanical positive reaction even after a week.

Further, all cases were tested at the same time against clear acrylic and other denture materials, and all positive results were very definite, no possible mechanical positive reactions being obtained with the other materials.

There were, as is usual in most series of patch tests, some anomalous results, and one in particular (Case 7) proved interesting. This patient gave a positive patch reaction to vulcanite dentures in January, 1952, at 48 hours. There appears to be little doubt about this, as he was demonstrated to a class of medical students at the time as a good example of a positive reaction. New dentures were made for him in acrylic, and his angular stomatitis cleared rapidly. He happened to have retained his old dentures, and in March, 1953, the patch test was repeated and proved to be negative at 96 hours.

Another patient (Case 3), who gave a delayed positive patch reaction to acrylic scrapings from his own dentures, failed to reproduce this when tested against acrylic applied direct to the skin. (These pieces were specially made and processed for two, four, six, and eight hours respectively.)

Although the evidence here is slender it is possible that some physical or electrochemical factor is concerned with these reactions and that the presence of saliva may in some way be bound up with this.

\section{Nature of the Sensitizing Material}

Lain (1932) states that the substance probably responsible for the sensitivity in vulcanite is vermilion, but as this material is rapidly becoming obsolete it was decided to investigate only the newer and more fashionable acrylic materials. Naturally, it was decided first to investigate the colouring material ; but the makers were unwilling to disclose its nature or to supply any for patch testing.

All patients were therefore patch-tested against coloured scrapings from their own dentures and clear acrylic scrapings. In only two cases (Nos. 6 and 46) was a positive result obtained to the coloured and a negative to the clear.

It was then decided to investigate a theory that sensitization is due to incomplete polymerization of monomer during the actual process of making the dental plates. This process consists in mixing liquid monomer and polymer powder into a dough, which is then heated in a mould, during which process the monomer polymerizes and the finished article results. This process probably depends on the presence of an organic peroxide or some such substance in the powder which acts as a source of free radicals which can initiate the polymerization of the added monomer. This reaction is slow at room temperature, but is nevertheless appreciable, since the mixture will set into a fairly hard material in a few days.

On heating, polymerization occurs more rapidly, the normal plate being processed at $100^{\circ} \mathrm{C}$. for about six hours.

Since polymerization takes place slowly at room temperature it seems unlikely that any monomer would be present, at least on the surface, even in an under-processed plate, after it had been made for a few weeks. However, it is possible that monomer could be trapped in the rigid polymer material in positions remote from initiator molecules, and it might thus be unchanged.

It seemed that the theory might be tenable if the monomer was a primary irritant or sensitizer, or if appreciable differences could be demonstrated in the amount of monomer present in acrylic material processed in different ways.

To test the first point all patients were patch-tested as a routine with monomer when being tested against denture materials, and in addition a control series of students and nursing staff were also tested. In no case did a patch test to monomer give a positive result even after 96 hours. It would therefore seem that liquid monomer is neither a primary irritant nor a potent sensitizer. 
TABLE I.-Summary of Results

\begin{tabular}{|c|c|c|c|c|c|c|c|c|c|c|c|}
\hline $\begin{array}{l}\text { Case } \\
\text { No. }\end{array}$ & $\begin{array}{c}\text { Age } \\
\text { (Years) }\end{array}$ & $\begin{array}{c}\text { Duration } \\
\text { of Con- } \\
\text { dition } \\
\text { (Years) }\end{array}$ & $\begin{array}{c}\text { Past } \\
\text { Medical History }\end{array}$ & $\begin{array}{l}\text { Worn } \\
\text { at } \\
\text { Night }\end{array}$ & $\begin{array}{l}\text { Material } \\
\text { of } \\
\text { Dentures }\end{array}$ & $\begin{array}{l}\text { Duration } \\
\text { of } \\
\text { Wearing } \\
\text { (Years) }\end{array}$ & $\begin{array}{l}\text { Mucosal } \\
\text { Lesions }\end{array}$ & $\begin{array}{l}\text { Patch Test } \\
\text { Results }\end{array}$ & $\begin{array}{l}\text { Ultimate } \\
\text { Diagnosis }\end{array}$ & Treatment & Result \\
\hline 1 & 45 & $2 / 12$ & Nervous dyspepsia $6 / 12$ & No & $\begin{array}{l}\text { Upper A. (6) } \\
\text { Lower V. 20 }\end{array}$ & 20 & No & Delayed + V. & $\begin{array}{c}\text { Sensitivity to } \\
\mathbf{V} \text {. }\end{array}$ & $\begin{array}{l}\text { A. Lowers } \\
\text { remade }\end{array}$ & $\begin{array}{l}\text { Improved, but no } \\
\text { completely cleared }\end{array}$ \\
\hline 2 & 47 & 3 & $\begin{array}{c}\text { Cholecystectomy (sto- } \\
\text { matitis followed al- } \\
\text { most immediately) }\end{array}$ & Yes & $\begin{array}{l}\text { A. }(20), \\
\text { V. } 2^{2}\end{array}$ & 20 & Yes & + A. $(96$ hrs. $)$ & $\begin{array}{l}\text { Sensitivity to } \\
\text { A. }\end{array}$ & $\begin{array}{l}\text { Remade. V. } \\
\text { O.B. } y^{\prime \prime}\end{array}$ & $\begin{array}{l}\text { Improved, but re- } \\
\text { lapsed. Had con- } \\
\text { siderable temporo- } \\
\text { mandibular } \\
\text { arthritis }\end{array}$ \\
\hline 3 & 38 & 8 & $\begin{array}{l}\text { P.O.W. in Germany at } \\
\text { onset. ? Vitamin defi- } \\
\text { ciency }\end{array}$ & No & B. & 14 & " & $+\underset{\text { Delayed +A. }}{\text { B., } 1952 .}$ & $\begin{array}{l}\text { Sensitivity to } \\
\text { B. and A. }\end{array}$ & $\begin{array}{c}\text { Remade } \\
\text { Oralite }\end{array}$ & $\begin{array}{l}\text { arthritis } \\
\text { Improved; but } \\
\text { relapsed }\end{array}$ \\
\hline 4 & 31 & 3 & $\begin{array}{l}\text { Low-grade hypochromic } \\
\text { anaemia }\end{array}$ & Yes & A. & $\begin{array}{c}14 \\
(\mathrm{New}, 1)\end{array}$ & $"$ & Neg. 96 hrs. & Malocclusion & O.B. & No change \\
\hline 5 & 72 & $2 / 12$ & $\begin{array}{l}\text { Constitutional eczema } \\
2 \text { years }\end{array}$ & No & A. & $\mid \begin{array}{c}\operatorname{lic} w, 1) \\
2\end{array}$ & $\begin{array}{l}\text { Very } \\
\text { minor }\end{array}$ & $\begin{array}{l}\text { Doubtful +A. } \\
96 \text { hrs. }\end{array}$ & $\begin{array}{r}\text { Sensitivity and } \\
\text { malocclusion }\end{array}$ & $\begin{array}{l}\text { Remade V; } \\
\text { O.B. } 3 / 16^{\prime}\end{array}$ & Cleared rapidly \\
\hline 6 & 59 & $1 / 12$. & $\begin{array}{l}\text { Constitutional eczema, } \\
\text { 1949. Hysterectomy, } \\
\text { 1942. Pruritus ani, } \\
1946 \text {. }\end{array}$ & " & A. & 17 & $\begin{array}{l}\text { only } \\
\text { No }\end{array}$ & $\begin{array}{l}+ \text { A. Neg. clear } \\
\text { A. and V. }\end{array}$ & $\begin{array}{l}\text { Sensitivity to } \\
\text { A. }\end{array}$ & $\begin{array}{l}\text { Remade V. } \\
\text { N.B. }\end{array}$ & Cleared \\
\hline 7 & 50 & $18 / 12$ & $\begin{array}{l}1946 \\
\text { Nil relevant }\end{array}$ & Yes & v. & 8 & Yes & $\begin{array}{l}+48 \text { hrs., } 1952 \\
\text { Neg. } 96 \text { hrs., }\end{array}$ & $\begin{array}{l}\text { Sensitivity to } \\
\text { V. }\end{array}$ & $\begin{array}{l}\text { Remade A. } \\
\text { O.B. } 3 / 16^{\prime \prime}\end{array}$ & " \\
\hline 8 & 31 & 2 & $\begin{array}{l}\text { Perforated gastric ulcer } \\
3 \text { years ago }\end{array}$ & ", & v. & 8 & $\begin{array}{l}\text { Slight } \\
\text { only }\end{array}$ & $\begin{array}{l}1953 \\
\text { Neg. }\end{array}$ & Malocclusion & O.B. $3 / 16^{\prime \prime}$ & $\because$ \\
\hline 9 & 55 & 2 & Nil releva & No & V. 20, A. 2 & 20 & No & $"$ & $"$ & $\begin{array}{l}\text { Remade A; } \\
\text { O.B. 3/16i }\end{array}$ & Improved \\
\hline 10 & 54 & 8 & $" \quad "$ & ” & A. & 7 & $"$ & $"$ & $?$ & $\begin{array}{l}X \text { rays } 100 \mathrm{r} \\
\times 3 \times 28\end{array}$ & Cleared \\
\hline 11 & 57 & $2 / 12$ & Psoriasis many years & " & v. & 7 & $"$ & $+48 \mathrm{hrs}$. & Sensitivity & $\begin{array}{l}\text { Remade A. } \\
\text { N.B. }\end{array}$ & Improved \\
\hline 12 & 48 & 10 & $\begin{array}{l}\text { Family history of stom- } \\
\text { atitis (see Case 23) }\end{array}$ & ", & A. & 10 & Yes & Neg. 96 hrs. & Familial & $\begin{array}{c}\text { Several new } \\
\text { sets made } \\
\text { N. and O.B }\end{array}$ & $\begin{array}{l}\text { Improved; but } \\
\text { relapsed }\end{array}$ \\
\hline 13 & 73 & 2 & Nil relevant & , & $\begin{array}{l}\text { Some ersatz } \\
\text { material. } \\
\text { V. } 1 \text { yr. }\end{array}$ & 4 & " & +V. 96 hrs. & $\begin{array}{l}\text { Sensitivity to } \\
\mathrm{V} \text {. }\end{array}$ & $\begin{array}{l}\text { Remade A. } \\
\text { N.B. }\end{array}$ & Cleared \\
\hline 14 & 35 & $1 / 12$ & $\begin{array}{l}\text { Seborrhoeic sycosis } \\
10 \text { years }\end{array}$ & Yes & v. & 25 & No & Neg. & $\begin{array}{l}\text { Infective } \\
\text { Malocclusion }\end{array}$ & O.B. $3 / 16^{\prime \prime}$ & $\begin{array}{l}\text { Cleared with tonsil- } \\
\text { litis. To have } \\
\text { tonsillectomy } \\
\text { Cleared }\end{array}$ \\
\hline 16 & 50 & 2 & $\begin{array}{l}\text { Cholecystectomy } 5 \\
\text { years ago }\end{array}$ & " & V. & 15 & Yes & $+V .48$ & $\begin{array}{c}\text { Sensitivity and } \\
\text { infection }\end{array}$ & $\begin{array}{l}\text { Remade A. } \\
\text { O.B. 3/16" }\end{array}$ & $\begin{array}{l}\text { Cleared; but relap- } \\
\text { ses with cold }\end{array}$ \\
\hline 17 & 45 & 3 & Nil relevant & ", & $\begin{array}{l}\text { V. (14) } \\
\text { A. (10) }\end{array}$ & 24 & No & Neg. & $\begin{array}{l}\text { Malocclusion } \\
\text { and } \\
\text { seborrhoeic }\end{array}$ & $\begin{array}{l}\text { Remade. } \\
\text { O.B. 3/16" }\end{array}$ & $\begin{array}{l}\text { Cleared; but re- } \\
\text { lapsed. Cleared } \\
\text { with ung. steroxin }\end{array}$ \\
\hline 18 & 36 & 1 & Malaria, 1922 & Yes & A. & 10 & $\underset{\text { Minor }}{\text { only }}$ & " & $\begin{array}{l}\text { seborrhoeic } \\
\text { Malocclusion }\end{array}$ & O.B. $1 / 16^{\prime \prime}$ & $\begin{array}{l}\text { with ung. steroxin } \\
\text { Cleared }\end{array}$ \\
\hline $\begin{array}{l}19 \\
20\end{array}$ & $\begin{array}{l}38 \\
60\end{array}$ & $\begin{array}{l}1 / 52 \\
3 / 12\end{array}$ & $\begin{array}{l}\text { Nil relevant } \\
" \quad "\end{array}$ & ", & v. & $\begin{array}{l}20 \\
15\end{array}$ & No" & $\begin{array}{l}\text { Doubtful+V. } \\
\text { Neg. }\end{array}$ & $\begin{array}{l}\text { Sensitivity } \\
\text { Malocclusion }\end{array}$ & $\begin{array}{l}\text { Remade A. } \\
\text { Remade A. } \\
\text { O.B. 3/16" }\end{array}$ & $"$ \\
\hline 21 & 62 & $2 / 12$ & Duodenal ulcer, 1943 & " & $\begin{array}{l}\text { A.-upper. } \\
\text { V.-lower }\end{array}$ & 40 & Yes & Neg. 96 & & $\begin{array}{l}\text { Remade A. } \\
\text { O.B. 3/16" }\end{array}$ & $\begin{array}{c}\text { Cleared and re- } \\
\text { lapsed }\end{array}$ \\
\hline 22 & 68 & 10 & $\begin{array}{l}\text { Congenital web in } \\
\text { throat. Operation, } \\
1943\end{array}$ & " & $\mathrm{v}$. & 40 & " & + V. 72 & Sensitivity & $\begin{array}{l}\text { Remade A: } \\
\text { N.B. }\end{array}$ & $\begin{array}{l}\text { No change, includ- } \\
\text { ing mouth lesions }\end{array}$ \\
\hline 23 & 47 & 20 & Crohn's disease at 38 & No & A. & $\begin{array}{r}19 \\
3\end{array}$ & No & Neg. & Malabsorption & N.B. & $\begin{array}{l}\text { Cleared after oper- } \\
\text { ation for Crohn's } \\
\text { disease }\end{array}$ \\
\hline 24 & 61 & $4 / 12$ & Nil relevant & Yes & V. & 15 & Yes & $\underset{96}{\text { Delayed }+V}$ & Sensitivity & $\begin{array}{l}\text { Remade A } \\
\text { O.B. 3/16" }\end{array}$ & Improved \\
\hline 25 & 60 & $6 / 12$ & Constitutional eczema & " & v. & 40 & No & Neg. & $\begin{array}{l}\text { Part of con- } \\
\text { stitutional } \\
\text { eczema }\end{array}$ & $\begin{array}{l}\text { Remade A. } \\
\text { N.B. }\end{array}$ & No change \\
\hline 26 & 43 & 2 & $\begin{array}{l}\text { Hysterectomy } 10 \text { years } \\
\text { ago }\end{array}$ & " & A. & 12 & Yes & , & Malocclusion & O.B. $3 / 16^{\prime \prime}$ & Cleared \\
\hline 27 & 22 & $8 / 12$ & Perlecche in childhood & - & & & & & " & Local only & $\begin{array}{c}\text { Cleared and } \\
\text { relapsed }\end{array}$ \\
\hline 28 & 57 & 4 & $\begin{array}{l}\text { Hysterectomy, 1931. } \\
\text { Thyroidectomy, 1930. } \\
\text { Lupus erythematosus, } \\
1934\end{array}$ & Yes & A. & 5 & Yes & Neg. 96 & ? Sensitivity & $\begin{array}{l}\text { Remade V. } \\
\text { N.B. }\end{array}$ & " \\
\hline 29 & 47 & $4 / 12$ & Nil relevant & No & A. & 8 & No & Neg. & Malocclusion & $\begin{array}{l}\text { Remade. } \\
\text { O.B. }\end{array}$ & Improved \\
\hline 30 & 36 & 2 & $\begin{array}{l}\text { Recurrent subluxation } \\
\text { temporo-mandibular } \\
\text { joint }\end{array}$ & Yes & V. & 17 & Yes & + V. 96 & Sensitivity & $\begin{array}{l}\text { Remade A. } \\
\text { N.B. }\end{array}$ & Cleared \\
\hline 31 & 73 & $6 / 12$ & Nil relevant & $\begin{array}{c}\text { Vari- } \\
\text { able }\end{array}$ & V. & 40 & No & Neg. & ? Sensitivity & $\begin{array}{l}\text { Remade A. } \\
\text { N.B. }\end{array}$ & $\begin{array}{l}\text { Cleared. Occa- } \\
\text { sional minor } \\
\text { relapse }\end{array}$ \\
\hline 32 & 46 & 2 & $\begin{array}{l}\text { Chronic perionychia } \\
13 \text { years }\end{array}$ & No & V. & $\begin{array}{r}23 \\
2\end{array}$ & Yes & " & Malocclusion & O.B. $3 / 16^{\prime \prime}$ & Improved \\
\hline 33 & 77 & 1 & Lichen planus & ", & V. & 20 & $"$ & Delayed $_{96}+\mathrm{V}$ & Sensitivity & $\begin{array}{l}\text { Remade A. } \\
\text { N.B. }\end{array}$ & $\begin{array}{l}\text { Cleared (no muco- } \\
\text { sal change) }\end{array}$ \\
\hline $\begin{array}{l}34 \\
35\end{array}$ & $\begin{array}{l}82 \\
52\end{array}$ & $\begin{array}{c}3 / 12 \\
3\end{array}$ & $\begin{array}{l}\text { Seborrhoeic dermatitis } \\
10 \text { years } \\
\text { Psoriasis } 25 \text { years. } \\
\text { Arthritis } 4 \text { years }\end{array}$ & ", & $\begin{array}{l}\text { V. } \\
\text { V. } \\
\text { A. }\end{array}$ & $\begin{array}{r}30 \\
12 \\
4\end{array}$ & $\begin{array}{l}\text { No } \\
\text { Yes } \\
\text { (patchy) }\end{array}$ & $\begin{array}{l}\text { Neg. } \\
\text { ", }\end{array}$ & $\begin{array}{l}\text { Part of seb. } \\
\text { derm. } \\
\text { Malocclusion }\end{array}$ & $\begin{array}{l}\text { Nil dental } \\
\text { O.B. } 3 / 16^{\prime \prime}\end{array}$ & $\begin{array}{l}\text { Cleared with gen- } \\
\text { eral condition } \\
\text { No change }\end{array}$ \\
\hline 36 & 73 & $2 / 12$ & Constitutional eczema & ", & V. & 4 & $\begin{array}{l}\text { Minor } \\
\text { only }\end{array}$ & ", & $\begin{array}{l}\text { Part of con- } \\
\text { stitutional } \\
\text { eczema }\end{array}$ & Nil dental & $\begin{array}{l}\text { Cleared with gen- } \\
\text { eral condition }\end{array}$ \\
\hline 37 & 56 & 4 & Nil relevant & Yes & V. & 30 & No & $"$ & Malocclusion & $\begin{array}{l}\text { Remade. } \\
\text { O.B. 3/16" }\end{array}$ & $\begin{array}{l}\text { Cleared and } \\
\text { relapsed }\end{array}$ \\
\hline 38 & 46 & 1 & $" \quad "$ & , & A. & 2 & " & ", & Infective & ntal & $\begin{array}{l}\text { Cleared with ung. } \\
\text { quinolor co. }\end{array}$ \\
\hline $\begin{array}{l}39 \\
40\end{array}$ & $\begin{array}{l}56 \\
31\end{array}$ & $\stackrel{2}{18 / 12}$ & Pleurisÿ with effusion & No & V. & $\begin{array}{r}38 \\
4\end{array}$ & $\begin{array}{l}\text { Yes } \\
\text { Yes } \\
\text { (L.P.- } \\
\text { like) }\end{array}$ & $\underset{96}{+ \text { Delayed }}+\mathbf{A}$ & $\begin{array}{c}\text { Sensitivity } \\
"\end{array}$ & $\begin{array}{l}\text { Remade A. } \\
\text { Remade V. }\end{array}$ & $\begin{array}{l}\text { Cleared } \\
\text { No change. Not a } \\
\text { case of angular } \\
\text { stomatitis, but in- } \\
\text { cluded because of } \\
\text { positive p a t c } \\
\text { test }\end{array}$ \\
\hline
\end{tabular}


TABLE I.-Summary of Results-continued

\begin{tabular}{|c|c|c|c|c|c|c|c|c|c|c|c|}
\hline $\begin{array}{l}\text { Case } \\
\text { No. }\end{array}$ & $\begin{array}{c}\text { Age } \\
\text { (Years) }\end{array}$ & $\begin{array}{c}\text { Duration } \\
\text { of Con- } \\
\text { dition } \\
\text { (Years) }\end{array}$ & $\begin{array}{c}\text { Past } \\
\text { Medical History }\end{array}$ & $\begin{array}{c}\text { Worn } \\
\text { at } \\
\text { Night }\end{array}$ & $\begin{array}{c}\text { Material } \\
\text { of } \\
\text { Dentures }\end{array}$ & $\begin{array}{l}\text { Duration } \\
\text { of } \\
\text { Wearing } \\
\text { (Years) }\end{array}$ & $\begin{array}{l}\text { Mucosal } \\
\text { Lesions }\end{array}$ & $\begin{array}{l}\text { Patch Test } \\
\text { Results }\end{array}$ & $\begin{array}{l}\text { Ultimate } \\
\text { Diagnosis }\end{array}$ & Treatment & Result \\
\hline 41 & 43 & 2 & $\begin{array}{l}\text { Parapsoriasis en plaque } \\
10 \text { years }\end{array}$ & Yes & A. & 2 & Yes & Neg. & & $\begin{array}{l}\text { Remade. } \\
\text { O.B. 3/16" }\end{array}$ & Cleared \\
\hline 42 & 61 & 7 & Thyroidectomy 10 years & No & V. & 20 & No & , & ? Sensitivity & $\begin{array}{l}\text { Remade A. } \\
\text { N.B. }\end{array}$ & $"$ \\
\hline 43 & 45 & 4 & Nil relevant & Yes & V. & $\begin{array}{r}11 \\
4\end{array}$ & Yes & " & ? Sensitivity & Remade V. & Improved \\
\hline 44 & 48 & $2 \frac{1}{2}$ & $" \quad$ " & No & V. & 15 & Slight & $\underset{96}{\text { Delayed }+V .}$ & & $\begin{array}{l}\text { Remade A. } \\
\text { N.B. }\end{array}$ & , \\
\hline 45 & 65 & $3 / 12$ & $\begin{array}{l}\text { Pernicious anaemia } \\
\text { since } 49\end{array}$ & " & V. & $\begin{array}{r}32 \\
5\end{array}$ & Yes & Neg. & Malocclusion & $\begin{array}{l}\text { Remade } \\
\text { O.B. } 3 / 16^{\prime \prime}\end{array}$ & Cleared \\
\hline 46 & 55 & 3 & Nil relevant & ", & A. & $\begin{array}{r}27 \\
5\end{array}$ & " & $\stackrel{?+\mathbf{A} .}{(\text { coloured) }}$ & ? Sensitivity & Remade V. & Cleared. N.B. \\
\hline
\end{tabular}

A. $=$ Acrylic. $\quad$ B. $=$ Bakelite. $\quad \mathrm{V}=$ Vulcanite. $\quad$ N.B. $=$ Normal bite. O.B. $=$ Opened bite

Secondly, investigation of the presence of monomer in denture material was carried out by submitting to a vacuum for several hours finely shaved particles of material made under varying conditions. Any loss of weight was taken to indicate loss of free monomer in the material.

\section{Investigation of Denture Material}

Sample I : Material processed at $100^{\circ} \mathrm{C}$. for two hours, and brought to $100^{\circ} \mathrm{C}$. slowly; age, several days. Sample II : Material brought to $100^{\circ}$ C. slowly and chilled suddenly without processing. Sample III : Material processed at $100^{\circ}$ C. for four hours; age, two days. Sample IV: As for Sample III, but aged five days.

TABLE II.-Results

\begin{tabular}{|c|c|c|c|c|}
\hline & $\underset{I}{\text { Sample }}$ & $\underset{\text { II }}{\text { Sample }}$ & $\underset{\text { III }}{\text { Sample }}$ & IV \\
\hline $\begin{array}{l}\text { Initial weight of sample (g.) } \\
\text { Loss of weight (g.) . } \\
\text { Percentage loss } \ldots\end{array}$ & $\begin{array}{l}0.0862 \\
0.0000 \\
0\end{array}$ & $\begin{array}{l}0.4277 \\
0.0200 \\
5\end{array}$ & $\begin{array}{l}0.2375 \\
0.0021 \\
0.9\end{array}$ & $\begin{array}{l}0.2592 \\
0.0016 \\
0.6\end{array}$ \\
\hline
\end{tabular}

The conclusions drawn from these results after allowance had been made for experimental error and other factors which might influence them were that, while it seems likely that some monomer is present in extreme cases of underprocessed material, it is impossible to say whether there is any in normally processed and aged material; but if any is present at all it is probably only a fraction of $1 \%$.

\section{Malocclusion}

Apparently, because it prevents discomfort when dentures are fitted, it is standard dental practice to close a patient's normal bite. This causes sagging of the mouth with accentuation of the angular creases. These tend to retain saliva and form an excellent nidus for organisms, increasing the likelihood of angular stomatitis. There is no doubt that this is a contributory factor in some cases; but many edentulous people never develop the condition.

In these series, results of opening the bite to remove the mechanical sagging proved rather conflicting. Some cases rapidly cleared completely, others cleared temporarily and relapsed, and one (Case 35 ) showed no improvement whatever.

The standard procedure here was to open the bite $3 / 16$ in. $(4.8 \mathrm{~mm}$.), but in many instances this proved impracticable mainly because of resultant discomfort in the nature of temporo-mandibular joint arthritis. To obviate this, it was decided to open the bite gradually $1 / 16$ in. $(1.6 \mathrm{~mm}$.) at a time every few months; but as yet there has not been time to assess the value of this technique.

It is to be noted that only one patient in this series (Case 29) did not wear dentures, and in this instance there was some malocclusion and apparently no other contributory cause, although the patient admitted to having had the habit of licking his lips in childhood. There was usually a quick response to any mild antiseptic applied locally, so that there was probably an infective cause.

\section{Results}

Table I gives the relevant findings in 46 cases. The nature of the study did not lend itself well to statistical analysis, and it was not thought profitable to pursue this. line of investigation.

Of the 46 cases treated, 22 cleared completely, 9 cleared but relapsed, 10 improved, and in 5 there was no change. Of those that were cleared, 18 had new dentures and 4 were given only local treatment.

\section{Summary and Conclusions}

A series of 46 cases of angular stomatitis associated with artificial dentures have been investigated.

There are several aetiological factors concerned in the production of angular stomatitis, and a full investigation of each case is necessary.

Patch tests to denture materials may be delayed, and patches should be left in situ for at least 96 hours.

The nature of the sensitizing material remains obscure, but may be connected with the colouring material.

Opening of the dental bite will result in the cure of some cases, but cannot be guaranteed to be permanent.

My thanks are due especially to Dr. J. T. Ingram for his helpful criticism and permission to use his cases in this investigation; to Drs. F. F. Helier and S. T. Anning, who also permitted me to use their cases; and to the staff of the dental school, without whose co-operation the practical work could not possibly have been carried out. Mr. McNaughton, of the chemistry department of the University of Leeds, also helped considerably in connexion with the investigation of incomplete polymerization of dental plates.

\section{REFERENCES}

Lain, E. S. (1932). Arch. Derm. Syph., Chicago, 25, 21 Vickers, H. R. (1949). British Medical Journal, 2, 1091

According to a report in the Manchester Guardian, it was made known at the Annual Conference of the National Institute of Houseworkers on May 18 that the Institute is now operating a programme for training hospital domestic workers. This scheme is being carried out in conjunction with the Leeds General Infirmary and the London Hospital. Domestic workers already employed in the hospital will receive a short part-time training, while a longer period of training is available for new employees between the ages of 15 and 18. A diploma will be awarded. The programme of training is being financed by grants from the Nuffield Provincial Hospitals Trust and the London Hospital free endowment funds. 